\title{
Multistable Pulse-like Solutions in a Parametrically Driven Ginzburg-Landau Equation
}

\author{
I.V. Barashenkov ${ }^{\star}$ and S. Cross ${ }^{\dagger}$ \\ Department of Mathematics and Applied Mathematics, \\ University of Cape Town, Rondebosch 7701, South Africa \\ Boris A. Malomed $\$$ \\ Department of Interdisciplinary Studies, Faculty of Engineering, Tel Aviv University, Tel Aviv 69978, Israel
}

(Dated: October 24, 2018)

\begin{abstract}
It is well known that pulse-like solutions of the cubic complex Ginzburg-Landau equation are unstable but can be stabilised by the addition of quintic terms. In this paper we explore an alternative mechanism where the role of the stabilising agent is played by the parametric driver. Our analysis is based on the numerical continuation of solutions in one of the parameters of the Ginzburg-Landau equation (the diffusion coefficient $c$ ), starting from the nonlinear Schrödinger limit (for which $c=0$ ). The continuation generates, recursively, a sequence of coexisting stable solutions with increasing number of humps. The sequence "converges" to a long pulse which can be interpreted as a bound state of two fronts with opposite polarities.
\end{abstract}

\section{INTRODUCTION}

A variety of nonequilibrium phenomena such as open flows in hydrodynamics, thermal convection in pure fluids and binary mixtures, processes in lasers and nucleation during the first-order phase transitions, can be modelled by the complex Ginzburg-Landau equations. (For review and reference, see e.g. 1, 2, 3, 4, 5].) Of primary importance are pulse-like solitary wave solutions, which represent localised structures widely observed in nonequilibrium systems. It is well known that in the cubic Ginzburg-Landau equation,

$$
i \psi_{t}+i \gamma \psi+(1-i c) \psi_{x x}+(2-i g)|\psi|^{2} \psi=0,
$$

solitary waves are unstable for all positive $c, \gamma$ and real $g$. In the $g>0$ case, however, they can be stabilised by the addition of quintic terms:

$$
i \psi_{t}+i \gamma \psi+(1-i c) \psi_{x x}+(2-i g)|\psi|^{2} \psi=-\left(q_{r}+i q_{i}\right)|\psi|^{4} \psi,
$$

with positive $q_{i}[6$, 7]. This example suggests that solitary pulses can be stable in a more general class of GinzburgLandau equations where the zero solution undergoes a subcritical (rather than supercritical) bifurcation to a flat nonzero solution [7]. In Eq.(2), the terms with $\gamma$ and $c$ account for linear homogeneous and nonhomogeneous losses, respectively, while the terms with $g$ and $q_{i}$ describe the cubic gain and quintic dissipation.

The present work deals with another equation of the Ginzburg-Landau type exhibiting the subcritical bifurcation, viz. the parametrically driven Ginzburg-Landau:

$$
i \psi_{t}+i \gamma \psi+(1-i c) \psi_{x x}+(2-i g)|\psi|^{2} \psi=h \psi^{*} e^{2 i \omega t} .
$$

Here, as in (1)-(2), the positive $c$ and $\gamma$ are the homogeneous loss and diffusion coefficients, respectively. The term with an asterisk (indicating the complex conjugation) represents the parametric driver. (The driver's amplitude $h$ can be chosen positive without loss of generality.) When $c=g=0$, Eq.(3) gives the parametrically driven damped nonlinear Schrödinger equation. This special case has been studied extensively; in particular, stable solitary waves [8] and their stable complexes [9] were found, and their bifurcations and supercritical dynamics analysed [10]. The objective of the present work is to advance beyond the nonlinear Schrödinger limit. We will still keep $g=0$ but allow for a nonzero diffusion coefficient, $c$. As we will see, even such a minimal generalisation gives rise to a new phenomenology of localised solutions which includes the multistability of pulses and pulse-front transitions.

Like the Ginzburg-Landau equations with intrinsic gain, the parametrically driven equation (36) arises in a wide range of physical applications. These include nonlinear Faraday resonance in vertically vibrated layers of water 11, 12, 13] and nonlinear lattices [14]; commensurate-incommensurate transitions in convective systems [15]; waves in nematic and cholesteric liquid crystals in rotating magnetic fields [16]; magnetisation waves in easy-plane ferromagnet exposed to microwave fields [8]; domain walls in the easy-axis ferromagnet near the Curie temperature [17] and in the easy-plane magnet in the stationary magnetic field [18]; nonlinear fiber lines with phase-sensitive amplification and mean-field models of degenerate optical parametric oscillators under continuous-wave pumping 19; pulsed optical parametric oscillators with spectral filtering and lasers with intracavity parametric amplification [20]. In most cases 
the models considered in literature are either purely diffusive $(c=\infty)$ [15, 16, 17, 20] or purely dispersive $(c=0)$ 8, 11, 12, 13, 18, 19]. However there are situations where it is crucial that $c$ be finite but nonzero. One physical phenomenon to which both diffusion and dispersion make essential contributions, is Faraday resonance in strongly dissipative media such as viscous fluids and granular materials 21]. Another situation where $0<c<\infty$, corresponds to nondegenerate optical parametric oscillators [22] and fiber-optic telecommunication links; in these contexts, the term $-i c \psi_{x x}$ represents spectral filtering in the spatial or temporal domain, respectively. In fact the applicability of Eq.(3) with nonzero $c$ and $g$ may happen to be even wider; it is commonly held that this equation provides a phenomenological description to a broad range of pattern-forming systems 23.

To study the solitary wave phenomenology introduced by taking the $\left(-i c \psi_{x x}\right)$-term into account, we will use the diffusion-free limit $(c=0)$ as a starting point, and perform the numerical continuation of analytical solutions available in that case, to nonzero $c$. The stability of solutions obtained in this way will also be studied numerically. We will show that "switching on" the diffusion gives rise to a sequence of stable multihumped pulses occurring in the vicinity of a certain particular value of the diffusion coefficient, $c_{\lim }=c_{\lim }(h, \gamma)$. The closer $c$ is to $c_{\lim }$, the greater is the number of multihump solutions coexisting at this $c$. The solutions with more than 5 or 6 humps describe a flat "plateau" (where $\psi$ is equal to the stable flat nonzero solution) sandwiched between two fronts of opposite polarity.

The paper is organised as follows. Section II deals mainly with spatially homogeneous solutions. In particular, we show that there is a stable flat nonzero solution for sufficiently large $c$, and this uniform solution can serve as a background for solitary waves. In section III we use perturbation-type arguments to demonstrate that both $\psi_{+}$ and $\psi_{-}$solitons are continuable in $c$ and in section [IV we construct the solutions with nonzero $c$ in the adiabatic approximation. The actual continuation is carried out numerically in section $\nabla$ where we also examine the stability of the continued solutions. Some additional insight into the bifurcation of stationary pulses is gained in section $\nabla \mathbf{I}$ Finally, section VII summarises results of this work.

We close this introduction by mentioning a recent publication 24] which was devoted to the study of the singlehumped solution of Eq.(3), by means of an averaging technique and direct numerical simulations. Neither the multistability of pulses nor the pulse-front transitions were dealt with in Ref. 24].

\section{EXISTENCE AND STABILITY INEQUALITIES}

The transformation $\psi(x, t) \rightarrow e^{-i \omega t} \psi(x, t)$ casts Eq. (3) in a 'standard' autonomous form

$$
i \psi_{t}+(1-i c) \psi_{x x}+2|\psi|^{2} \psi-\psi=h \psi^{*}-i \gamma \psi
$$

where we set $g=0$ and rescaled $t$ so that $\omega=1$. This is the representation that we are going to work with in what follows.

The equation (4) has three time-independent spatially uniform solutions, or 'flat backgrounds', for short. (We do not distinguish between solutions different only in the overall sign here.) One flat solution is $\psi_{\text {flat }}=0$; it will be central for this work where we are focussing on solutions decaying to zero at infinities. The other two flat solutions are given by

$$
\psi_{\text {flat }}=\Psi_{ \pm}^{(0)} \equiv\left(A_{ \pm} / \sqrt{2}\right) e^{-i \Theta_{ \pm}}
$$

where

$$
A_{ \pm}=\sqrt{1 \pm \sqrt{h^{2}-\gamma^{2}}}, \quad 2 \Theta_{ \pm}=\arccos \left( \pm \sqrt{1-\gamma^{2} / h^{2}}\right)
$$

Note that these solutions do not depend on $c$.

\section{A. Stability of spatially uniform solutions}

One can easily check that the zero solution is stable as long as $h<\sqrt{1+\gamma^{2}}$, irrespectively of the value of $c \geq 0$. The analysis of the flat nonzero solutions is somewhat more laborious.

Linearising Eq. (4) about $\psi_{\text {flat }}=\Psi_{ \pm}^{(0)}$, and assuming a perturbation $\delta \psi \propto \exp [i(\omega t-k x)]$ yields the dispersion relation

$$
i \omega=-\left(c k^{2}+\gamma\right) \pm i \sqrt{Z}
$$


where

$$
Z=\left(1-2 A^{2}+k^{2}\right)^{2}+A^{2}\left(A^{2}-2\right)-h^{2} .
$$

(Here $A$ stands for $A_{+}$or $A_{-}$, depending on which solution we are linearizing about.) The flat solution is stable iff $\operatorname{Re}(i \omega) \leq 0$, i.e., when $Z \geq-\left(c k^{2}+\gamma\right)^{2}$, for all real $k$. The latter condition amounts to an inequality

$$
\left(1+c^{2}\right) s^{2}+2\left(1-2 A^{2}+\gamma c\right) s+4 A^{2}\left(A^{2}-1\right) \geq 0
$$

where $s$ stands for $k^{2}$.

Let, first, $\psi=\Psi_{-}^{(0)}$. Since $A_{-}^{2}<1$, the inequality (9) does not hold for $k=0$; hence the 'low' background $\Psi_{-}^{(0)}$ is always unstable.

Let now $\psi=\Psi_{+}^{(0)}$, the 'high' background. The quadratic expression in (9) will be positive for all $s \geq 0$ if either its two roots $s_{1}$ and $s_{2}$ are both real negative or complex. (Note that we cannot have two real roots of opposite signs as the quadratic's constant term, $4 A^{2}\left(A^{2}-1\right)$, is always positive for $\psi=\Psi_{+}^{(0)}$.) Whether the roots are real or complex is determined by the discriminant of the quadratic (9) which can be written as

$$
\mathcal{D}=\left[\gamma^{2}-4 A_{+}^{2}\left(A_{+}^{2}-1\right)\right]\left(c-c_{+}\right)\left(c-c_{-}\right) .
$$

Here we have introduced

$$
c_{ \pm}=\frac{\left(2 A_{+}^{2}-1\right) \gamma \pm 2 A_{+} \sqrt{\left(A_{+}^{2}-1\right)\left(1+\gamma^{2}\right)}}{\gamma^{2}-4 A_{+}^{2}\left(A_{+}^{2}-1\right)} .
$$

We need to consider two cases. Assume, first, that $4 A_{+}^{2}\left(A_{+}^{2}-1\right)<\gamma^{2}$. In this case the discriminant is negative (and hence, the roots $s_{1,2}$ are complex) provided $c$ lies in the interval $c_{-}<c<c_{+}$. On the other hand, the roots $s_{1,2}$ are real negative in this case if $\mathcal{D}$ is $\geq 0$ and the coefficient in front of the middle term in (9) is positive:

$$
c>c_{0} \equiv\left(2 A_{+}^{2}-1\right) / \gamma .
$$

Since in the case at hand we have $0<c_{-}<c_{0}<c_{+}$, the union of the above two 'stable' regions, $c_{-}<c<c_{+}$and $c>c_{+}$, with the endpoints included, is simply $c \geq c_{-}$.

The second case is defined by inequality $4 A_{+}^{2}\left(A_{+}^{2}-1\right)>\gamma^{2}$. Here we have $c_{+}<0<c_{-}<c_{0}$ and the quadratic (9) cannot have negative real roots as the region (11) does not overlap with the region where $\mathcal{D} \geq 0$. However, it can have complex roots - provided $c>c_{-}$.

Thus we arrive at a simple stability criterion for the flat nonzero solution $\Psi_{+}^{(0)}$, valid for all $h$ and $\gamma$ :

$$
c \geq c_{-}(h, \gamma)
$$

with $c_{-}$as in (10). Note that the stability threshold $c_{-}(h, \gamma)$ is always strictly positive. This implies, in particular, that the solution $\Psi_{+}^{(0)}$ is always unstable in the case $c=0$. (This fact has already been mentioned in the literature 25].) Therefore the 'focusing', or 'attractive', damped-driven Schrödinger equation (Eq. (4) with $c=0$ ) does not have stable flat backgrounds except the trivial one, $\psi=0$. The analysis of localised solutions over flat nonzero backgrounds becomes meaningful only within the full Ginzburg-Landau equation, i.e. Eq.(4) with positive $c$.

\section{B. The flat solutions as backgrounds to solitary waves}

No less important is the question of when a flat solution can serve as an asymptotic value to a localised waveform - in other words, when is spatial decay to the flat solution possible. To find the corresponding criterion, we set $\omega=0$ in equation (7). This results in a quadratic equation

$$
\left(1+c^{2}\right) s^{2}-2\left(2 A^{2}-1-\gamma c\right) s+4 A^{2}\left(A^{2}-1\right)=0
$$

where $s=k^{2}$. The spatial decay to a flat background is possible unless both roots of Eq. (13), $s_{1}$ and $s_{2}$, are positive.

In the case of the $\Psi_{-}^{(0)}$ background, the discriminant of equation (13) is positive while the constant term is negative, whence $s_{1}>0$ and $s_{2}<0$. Consequently, the decay to $\Psi_{-}^{(0)}$ may occur for all values of $h, \gamma$ and $c$. (This fact is of little importance, however, since we have just shown that this background is always unstable.) 
In the case of the $\Psi_{+}^{(0)}$ solution, we may have two positive roots - provided $\mathcal{D}>0$ and $c<c_{0}$, with $c_{0}$ as in (11). Following the steps in the previous subsection, one can readily show that this situation occurs only if $0<c<c_{-}$, with $c_{-}$as in (10). Thus the stable constant solutions, defined by inequality (12), can always serve as backgrounds to fronts and pulses. We will come across localised solutions over nonvanishing backgrounds in section $\nabla$ below.

Finally, to examine the case of the zero background, we set both $A=0$ and $\omega=0$ in equation (7). This yields

$$
\left(1+c^{2}\right) s^{2}+2(\gamma c+1) s+\gamma^{2}-h^{2}=0
$$

where, as before, $s=k^{2}$. Since the middle term in (14) has a positive coefficient, we have $s_{1}+s_{2}<0$, which means that the situation where both $s_{1}$ and $s_{2}$ are positive, can never occur in this case. Thus the decay to the zero background is possible for all $h, \gamma$ and $c$.

\section{The threshold driving strength for pulses}

Our last result in this section concerns the range of driving strengths which can support localised solutions in the presence of dissipation. Multiplying Eq. (4) by $\psi^{*}$ and subtracting the complex conjugate of the resulting equation gives what would be a local conservation law of the number of particles if $c, h$ and $\gamma$ were equal to zero:

$$
\begin{array}{r}
i \partial_{t}|\psi|^{2}+\left(\psi_{x} \psi^{*}-\psi_{x}^{*} \psi\right)_{x} \\
=h\left[\left(\psi^{*}\right)^{2}-\psi^{2}\right]-2 i \gamma|\psi|^{2}+i c\left(\psi_{x x} \psi^{*}+\psi_{x x}^{*} \psi\right) .
\end{array}
$$

Assuming that $\psi$ and $\psi_{x} \rightarrow 0$ as $|x| \rightarrow \infty$ and integrating (15) over the real line, we get

$$
\frac{d}{d t} \int|\psi|^{2} d x=2 \int|\psi|^{2}[h \sin (2 \chi)-\gamma] d x-2 c \int\left|\psi_{x}\right|^{2} d x,
$$

where we have denoted $-\chi(x, t)$ the phase of the complex field $\psi: \psi=|\psi| e^{-i \chi}$. Let $h<\gamma($ and remember that $c>0$ ). In this case it follows from (16) that the time derivative of $\int|\psi|^{2} d x$ remains negative at all times. Hence as $t \rightarrow \infty$, $\psi(x, t) \rightarrow 0$ for all $x$. No stationary, time-periodic, quasiperiodic or chaotic solutions, decaying to zero as $|x| \rightarrow \infty$, can arise if $h<\gamma$. We will make use of this simple criterion in what follows.

\section{CONTINUABILITY OF THE TWO NLS SOLITONS}

In the limit $c=0$, Eq.(4) becomes the parametrically driven damped nonlinear Schrödinger (NLS) equation, which has exact time-independent solitary wave solutions [8, 11] of the form

$$
\psi_{ \pm}(x, t)=A_{ \pm} e^{-i \Theta_{ \pm}} \operatorname{sech}\left(A_{ \pm} x\right),
$$

with $A_{ \pm}$and $\Theta_{ \pm}$as in (6). The solution $\psi_{-}$is unstable for all $h$ and $\gamma$, while $\psi_{+}$is stable in a certain parameter region [8].

The purpose of this section is to show that the solitary pulse solutions $\psi_{ \pm}$of the NLS equation persist for $c \neq 0$. We restrict ourselves to stationary solutions $\left(\psi_{t}=0\right)$. Writing $\psi=\phi e^{-i \Theta}$ with $\Theta$ a constant phase to be chosen later, Eq.(4) becomes an ordinary differential equation for $\phi$ :

$$
(1-i c) \phi_{x x}+2|\phi|^{2} \phi-(1-i \gamma) \phi=h \phi^{*} e^{2 i \Theta}
$$

To find out whether solutions available at $c=0$ can be continued to nonzero $c$, we expand $\phi$ in power series $\phi=\phi_{0}+c \phi_{1}+c^{2} \phi_{2}+\ldots$, substitute into Eq. (18) and match like powers of $c$. It is convenient to choose $\Theta$ to be $\Theta_{+}$ in the case of the soliton $\psi_{+}$, and $\Theta_{-}$for $\psi_{-}$; this choice makes $\phi_{0}$ real. Matching terms linear in $c$ and decomposing $\phi_{1}$ into its real and imaginary part $\left(\phi_{1}=u+i v\right)$, yields an equation

$$
L_{ \pm}\left(\begin{array}{l}
u \\
v
\end{array}\right)=\left(\begin{array}{c}
0 \\
-\phi_{0}^{\prime \prime}
\end{array}\right)
$$

where the primes stand for the derivatives in $x$ and the operators $L_{ \pm}$are defined by

$$
L_{ \pm}=\left(\begin{array}{cc}
-\partial_{x}^{2}+1-6 \phi_{0}^{2}+h \cos \left(2 \Theta_{ \pm}\right) & 2 \gamma \\
0 & -\partial_{x}^{2}+1-2 \phi_{0}^{2}-h \cos \left(2 \Theta_{ \pm}\right)
\end{array}\right)
$$


(The subscripts + and - indicate whether we are checking the continuability of $\psi_{+}$or $\psi_{-}$. .)

According to Fredholm's alternative, Eq.(19) has bounded solutions if and only if its right-hand side is orthogonal to the kernel of the adjoint operator $L_{ \pm}^{\dagger}$. Transforming to $\xi \equiv A_{+} x$ and $\xi \equiv A_{-} x$ in the case of the $\psi_{+}$and $\psi_{-}$ solution, respectively, the equation for the zero modes spanning $\operatorname{ker} L_{ \pm}^{\dagger}$ becomes

$$
\left(\begin{array}{cc}
L_{1} & 0 \\
2 \gamma & L_{0}-\epsilon_{ \pm}
\end{array}\right)\left(\begin{array}{l}
f \\
g
\end{array}\right)=0
$$

where $f=f(\xi), g=g(\xi)$,

$$
\epsilon_{ \pm} \equiv 2 \frac{A_{ \pm}^{2}-1}{A_{ \pm}^{2}}= \pm 2 \frac{\sqrt{h^{2}-\gamma^{2}}}{1 \pm \sqrt{h^{2}-\gamma^{2}}}
$$

and we have introduced the standard Pöschl-Teller operators with familiar spectral properties:

$$
L_{0}=-\partial_{\xi}^{2}+1-2 \operatorname{sech}^{2} \xi, \quad L_{1}=-\partial_{\xi}^{2}+1-6 \operatorname{sech}^{2} \xi
$$

The only discrete eigenvalue of $L_{0}$ is $E_{0}=0$, while the continuous spectrum occupies the semiaxis $E \geq 1$. Therefore, the operator $\left(L_{0}-\epsilon_{ \pm}\right)$is invertible as long as $\epsilon_{ \pm}<1$ and $\epsilon_{ \pm} \neq 0$. Assuming that the zero background is stable (i.e., assuming that $\left.h^{2}<1+\gamma^{2}\right)$, the quantity $\epsilon_{+}$is indeed less than one but greater than zero. On the other hand, the $\epsilon_{-}$ is negative. (An exception is the point $h=\gamma$, where we have $\epsilon_{ \pm}=0$.) Thus we conclude that $\left(L_{0}-\epsilon_{ \pm}\right)$is invertible except in the special case $h=\gamma$. (However, even in this special case the operator $L_{0}$ is invertible on odd functions because the eigenfunction sech $\xi$ associated with the zero eigenvalue is even.) Consequently, $f(\xi)$ cannot be equal to zero for $h \neq \gamma$-otherwise the bottom equation in (21) would imply that $g(\xi)=0$, too. Fortunately, the operator $L_{1}$ does have a zero eigenvalue, and therefore $f(\xi)$ can be a nonzero multiple of the corresponding eigenfunction (which is $\tanh \xi \operatorname{sech} \xi$.)

Thus in the case $h \neq \gamma$ the kernel of $L_{ \pm}^{\dagger}$ is spanned by just one zero mode, namely

$$
\left(\begin{array}{l}
f_{1} \\
g_{1}
\end{array}\right)=\left(\begin{array}{c}
\tanh \xi \operatorname{sech} \xi \\
-2 \gamma\left(L_{0}-\epsilon_{ \pm}\right)^{-1}(\tanh \xi \operatorname{sech} \xi)
\end{array}\right) .
$$

On the other hand, when $h=\gamma$, the dimension of the kernel space is two. Firstly, the zero mode (22) persists for $\epsilon_{ \pm}=0$ as the operator $L_{0}^{-1}$ is defined on odd functions. Secondly, there is another zero mode given by

$$
\left(\begin{array}{l}
f_{2} \\
g_{2}
\end{array}\right)=\left(\begin{array}{c}
0 \\
\operatorname{sech} \xi
\end{array}\right)
$$

It is obvious that the vector-function (22), whose both components are odd functions of $\xi$, is orthogonal to the right-hand side of Eq.(19) - which is even. Hence Eq.(19) is solvable for $h \neq \gamma$. It is also easy to check that the mode (23) is not orthogonal to the right-hand side of (19) and so the solvability condition is not satisfied for $h=\gamma$.

Thus, having started from the two nonlinear Schrödinger solitons, we constructed two one-pulse solutions of the Ginzburg-Landau equation to within the first order in the small parameter $c: \quad \psi=\psi_{ \pm}+c e^{-i \Theta_{ \pm}}(u+i v)+\ldots$ Consequently, we expect to be able to continue the Schrödinger solitons $\psi_{ \pm}$into the region $c \neq 0$ (provided that $h \neq \gamma$.) This expectation is born out by results displayed in section $\nabla$ below, which present an outcome of the numerical continuation of $\psi_{ \pm}$in $c$.

\section{ADIABATIC APPROXIMATION}

Before attempting the full-scale numerical continuation, it is instructive to construct approximate solutions. Our approximation will be valid for small $c$ and exploit the fact that when $c=0$, the stationary pulse-like solutions of equation (44) have the form

$$
\psi=a \operatorname{sech}(a x) e^{-i \theta}
$$

where $a=A_{ \pm}$and $\theta=\Theta_{ \pm}$are constants defined by Eqs.(6). For $c$ small nonzero, approximate solutions can be sought for by assuming that $\psi$ retains the form (24), but $a$ and $\theta$ become slowly varying functions of $t$. 
To obtain an expression for $\dot{a}$ (the overdot stands for the derivative in $t$ ), we substitute the ansatz (24) into (15). Integrating over $x$ and using the boundary conditions $\psi_{x} \rightarrow 0$ as $|x| \rightarrow \infty$ produces an evolution equation for the pulse's amplitude:

$$
\dot{a}=2 a\left(h \sin 2 \theta-\gamma-\tilde{c} a^{2}\right),
$$

where $\tilde{c} \equiv c / 3$. An equation for the pulse's phase arises by multiplying (44) by $\psi^{*}$, adding with its complex conjugate, substituting (24) for $\psi$ and integrating over $x$ :

$$
\dot{\theta}=h \cos 2 \theta+1-a^{2} .
$$

Fixed points of the system (25) and (26) correspond to stationary solutions of (4). These can be easily found explicitly: Eliminating $\theta$ from the stationary system

$$
h \sin (2 \theta)=\tilde{c} a^{2}+\gamma, \quad h \cos (2 \theta)=a^{2}-1,
$$

produces a quadratic equation

$$
\left(1+\tilde{c}^{2}\right) a^{4}+2(\gamma \tilde{c}-1) a^{2}+1+\gamma^{2}-h^{2}=0
$$

with roots

$$
a_{ \pm}^{2}=\frac{(1-\gamma \tilde{c}) \pm \sqrt{\left(h^{2}-1\right) \tilde{c}^{2}-2 \gamma \tilde{c}+h^{2}-\gamma^{2}}}{1+\tilde{c}^{2}} .
$$

The corresponding $\theta_{ \pm}$are defined by their sine and cosine in (27).

It is not difficult to determine when the roots (29) are real and positive. We assume that the zero background solution of Eq.(41) is stable, i.e. the constant term in (28) is positive. Hence if the roots are real, they are of the same sign, and this sign is opposite to that of the middle term in (28). Therefore we have two positive roots provided the discriminant

$$
\mathcal{D}=\left(h^{2}-1\right) \tilde{c}^{2}-2 \gamma \tilde{c}+h^{2}-\gamma^{2} \equiv\left(h^{2}-1\right)\left(\tilde{c}-\tilde{c_{1}}\right)\left(\tilde{c}-\tilde{c_{2}}\right)
$$

is nonnegative, and, at the same time, the inequality $\gamma \tilde{c}<1$ holds true. In (30) we have introduced

$$
\tilde{c}_{1,2}=\frac{\gamma \pm h \sqrt{1+\gamma^{2}-h^{2}}}{h^{2}-1}
$$

where the + corresponds to $\tilde{c}_{1}$ and - to $\tilde{c}_{2}$.

It is straightforward to verify that for small $h, h^{2}<1$, we have $\tilde{c_{1}}<0<\tilde{c_{2}}<\frac{1}{\gamma}$, while for larger $h, h^{2}>1$, we have $0<\tilde{c_{2}}<\frac{1}{\gamma}<\tilde{c_{1}}$. (Here we have made use of the inequality $h>\gamma$; as proved in section ЩC no stationary localised solutions exist for $h<\gamma$.) Therefore, the region of $c$ values where the roots of (28) are positive, is given by the inequality $\tilde{c}<\tilde{c_{2}}(h, \gamma)$ - for all $h$.

Thus we conclude that the adiabatic equations have two stationary points for $c<3 \tilde{c_{2}}(h, \gamma)$, and none for $c>3 \tilde{c_{2}}$. We complete the adiabatic analysis by classifying their stability and bifurcation.

Linearising equations (26) about the stationary points and assuming small perturbations of the form $\delta a=C_{1} e^{2 \lambda t}$ and $\delta \theta=C_{2} e^{2 \lambda t}$, yields a characteristic equation

$$
\lambda^{2}+\left(\gamma+c a^{2}\right) \lambda+2 h a^{2} \cos (2 \theta)-h \sin (2 \theta)\left[h \sin (2 \theta)-\gamma-c a^{2}\right]=0 .
$$

Since the coefficient in front of the middle term in this equation is positive, either its two roots are complex with negative real parts, or we have two real roots, of which one is negative. The case when the second root is positive (and hence the fixed point is unstable) occurs if the constant term is negative. Conversely, if the constant term is nonnegative:

$$
2 h a^{2} \cos (2 \theta)-h \sin (2 \theta)\left[h \sin (2 \theta)-\gamma-c a^{2}\right] \geq 0,
$$

the fixed point is stable. Simplifying Eq.(32), we get the stability condition in the form

$$
a^{2} \geq \frac{1-\gamma \tilde{c}}{1+\tilde{c}^{2}}
$$




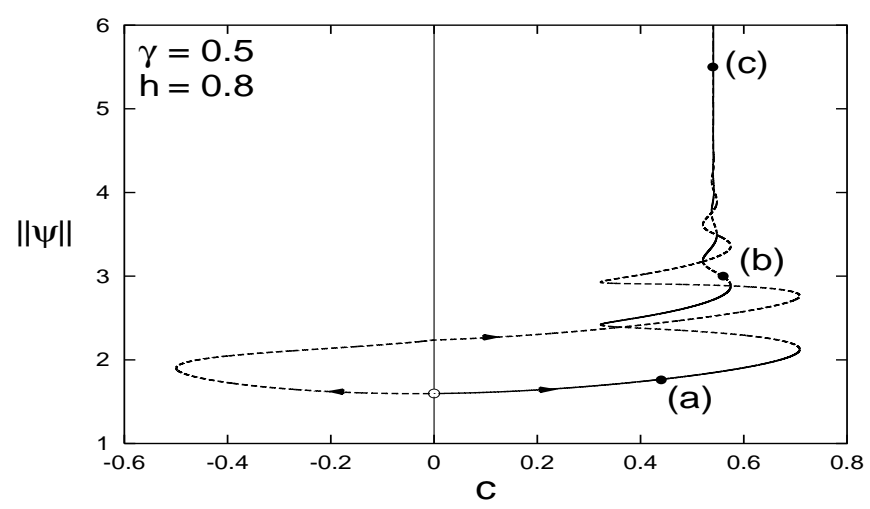

FIG. 1: The bifurcation diagram displaying the Sobolev norm versus the diffusion coefficient, for the solitary pulse obtained by the continuation of the stable soliton $\psi_{+}$in $c$. The continuation starts from a point on the $(c=0)$-axis, marked by an open circle. The arrows indicate the directions of continuation and are only added for reference purposes. Solutions at points marked by the black dots (a), (b) and (c) are shown in Figure 2] The solid lines correspond to stable and dashed curves to unstable branches.

Comparing this to the expressions for $a_{ \pm}$in (29), we conclude that for all $c, h$ and $\gamma$, the points $\left(a_{+}, \theta_{+}\right)$and $\left(a_{-}, \theta_{-}\right)$ are a stable node and saddle, respectively.

The two fixed points come into being through a saddle-node bifurcation which occurs as the diffusion coefficient $c$ is decreased past $c=3 \tilde{c}_{2}$ for the fixed $h$ and $\gamma$ or, alternatively, as the driving amplitude $h$ is increased for the fixed dissipation coefficients $c$ and $\gamma$. One can easily find the bifurcation value of $h$, at which two complex roots of the quadratic equation (28) converge on the positive real axis. (Here we are assuming that $\tilde{c}$ is smaller than $\frac{1}{\gamma}$.) Writing the discriminant (30) as

$$
\mathcal{D}=\left(\tilde{c}^{2}+1\right)\left[h^{2}-\frac{(\tilde{c}+\gamma)^{2}}{\tilde{c}^{2}+1}\right]
$$

the threshold driving strength is given by

$$
h_{\mathrm{ad}}=\frac{\tilde{c}+\gamma}{\sqrt{\tilde{c}^{2}+1}}
$$

where the subscript "ad" serves to remind that Eq.(33) was obtained in the adiabatic approximation. It is important to emphasise that this formula is valid only for small $\tilde{c}$. Equivalently, the expression (31) for the turning point $\tilde{c}_{2}$ is valid only for $h$ close to $\gamma$.

\section{NUMERICAL CONTINUATION AND STABILITY ANALYSIS}

In this section we describe the bifurcation diagram obtained by the numerical continuation of the solitons $\psi_{ \pm}$in the parameter $c$. The diagram is presented in Fig 1 and displays the Sobolev norm of the solution,

$$
\|\psi\|=\sqrt{\int\left(\left|\psi_{x}\right|^{2}+|\psi|^{2}\right) d x}
$$

as a function of $c$.

\section{A. The method}

For stationary solutions, $\psi=\psi(x)$, equation (4) reduces to an ordinary differential equation

$$
(1-i c) \psi_{x x}+2|\psi|^{2} \psi-(1-i \gamma) \psi=h \psi^{*} .
$$

For the numerical continuation of solutions to Eq. (34) we utilised the AUTO97 software package [26]. The infinite line was approximated by a finite interval $(-L, L)$, with $L=100$, and the boundary conditions $\psi( \pm L)=0$. The 
tolerance of the computation (the grid norm of the difference between the right and left-hand side of (34)) was set to be $10^{-7}$.

The solid and broken curves in Fig 1 represent stable and unstable branches, respectively. The stability was examined by linearising Eq.(4) about the corresponding stationary solution. Choosing the small perturbation in the form $\delta \psi(x, t)=[u(x)+i v(x)] e^{\lambda t}$, with real $u$ and $v$, we arrive at an eigenvalue problem

$$
\mathcal{H}\left(\begin{array}{l}
u \\
v
\end{array}\right)=\lambda J\left(\begin{array}{l}
u \\
v
\end{array}\right)
$$

where

$$
\mathcal{H}=\left(\begin{array}{lr}
-\partial_{x}^{2}+1+h-6 \mathcal{R}^{2}-2 \mathcal{I}^{2} & -c \partial_{x}^{2}+\gamma-4 \mathcal{R} \mathcal{I} \\
c \partial_{x}^{2}-\gamma-4 \mathcal{R} \mathcal{I} & -\partial_{x}^{2}+1-h-2 \mathcal{R}^{2}-6 \mathcal{I}^{2}
\end{array}\right)
$$

and

$$
J=\left(\begin{array}{rr}
0 & -1 \\
1 & 0
\end{array}\right)
$$

The $\mathcal{R}$ and $\mathcal{I}$ are the real and imaginary parts of the solution: $\psi=\mathcal{R}+i \mathcal{I}$. We solved the eigenvalue problem by expanding $u$ and $v$ over 500 Fourier modes in the interval $(-100,100)$.

\section{B. Continuation and stability}

The continuation in $c$ was performed for fixed values of $h$ and $\gamma$. We selected $\gamma=0.5$ and $h=0.8$; for these $h$ and $\gamma$ the nonlinear Schrödinger soliton $\psi_{+}$is stable [8]. Before proceeding to the $\psi_{+}$soliton, however, we briefly deal with the $\psi_{-}$-case. In agreement with predictions of section III the soliton $\psi_{\text {- was found to persist both for } c<0}$ and $c>0$. We were in fact able to continue it indefinitely without encountering any obstacles in both directions. As $c \rightarrow \infty$, the width of the localised solution of (34) grows and the solution tends to $\phi_{\gamma, h}(x / \sqrt{c})$, where $\phi_{\gamma, h}(X)$ stands for a pulse-shaped solution of equation

$$
-i \phi_{X X}+2|\phi|^{2} \phi-(1-i \gamma) \phi=h \phi^{*}
$$

In a similar way, as $c \rightarrow-\infty$, the solution tends to $\phi_{-\gamma, h}^{*}(x / \sqrt{-c})$. When continued to $c>0$, the pulse remained unstable for all $c$, with a single positive eigenvalue in its spectrum. (As for the negative- $c$ region, all solutions there are a priori unstable against continuous spectrum perturbations with arbitrarily large $\operatorname{Re} \lambda$.)

The continuation of the $\psi_{+}$soliton proved to be more rewarding from the stability viewpoint. The corresponding bifurcation diagram is displayed in Fig 1 As with the soliton $\psi_{-}$, the $\psi_{+}$persists both for $c>0$ and $c<0-$ in agreement with section III Continuing into the $c>0$ region, we have found that the solution gradually changes its shape, with the hump in the imaginary part splitting into two (see Fig 2(a)). At $c=0.7$ the branch turns back (Fig 1). Note that the turning point occurs for $c$ much smaller than $3 \tilde{c}_{2}=1.04$, the saddle-node bifurcation point predicted by the adiabatic analysis. What is more important, the $\psi_{+}$solution turns not into the $\psi_{-}$(as the adiabatic approach predicted) but into some other solution which has two well-separated humps in the imaginary part. The discrepancy is not surprising as the adiabatic approximation is valid only for small $c$, where the shape of the solution is still well reproduced by the single-hump constant-phase trial function (24). In the entire stretch between $c=0$ and the turning point, the solution remains stable.

Continuing this branch further, additional humps are added as the solution passes a sequence of turning points. Each pass of a turning point results in the creation of a new hump in the middle of the solitary wave. As we move along the branch, the distance between successive turning points (the difference between the corresponding values of c) becomes smaller and the new humps come with smaller amplitudes. As a result, a long plateau is formed which keeps on expanding as we continue the branch (Fig 2 (c)). The broadening plateau accounts for the vertical segment of the curve in Fig [ If the bifurcation parameter $c$ is seen as a function of the Sobolev norm $\|\psi\|$ (that is, if we turn Fig 1 by $\left.90^{\circ}\right)$, the curve $c(\|\psi\|)$ has the form of a decaying oscillation.

The stability of the solution alternates at each successive turning point. These changes are due to a single real eigenvalue which moves back and forth through the origin on the real line. (At the turning points the eigenvalue is right at the origin, of course.) The lengths of the incursions this eigenvalue makes into the positive and negative real lines decrease with each new turning point until the eigenvalue becomes indistinguishable from zero. Therefore the branch becomes (neutrally) stable sufficiently 'high up' in $\|\psi\|$ in Fig 1 (i.e. for sufficiently long plateaus.) 

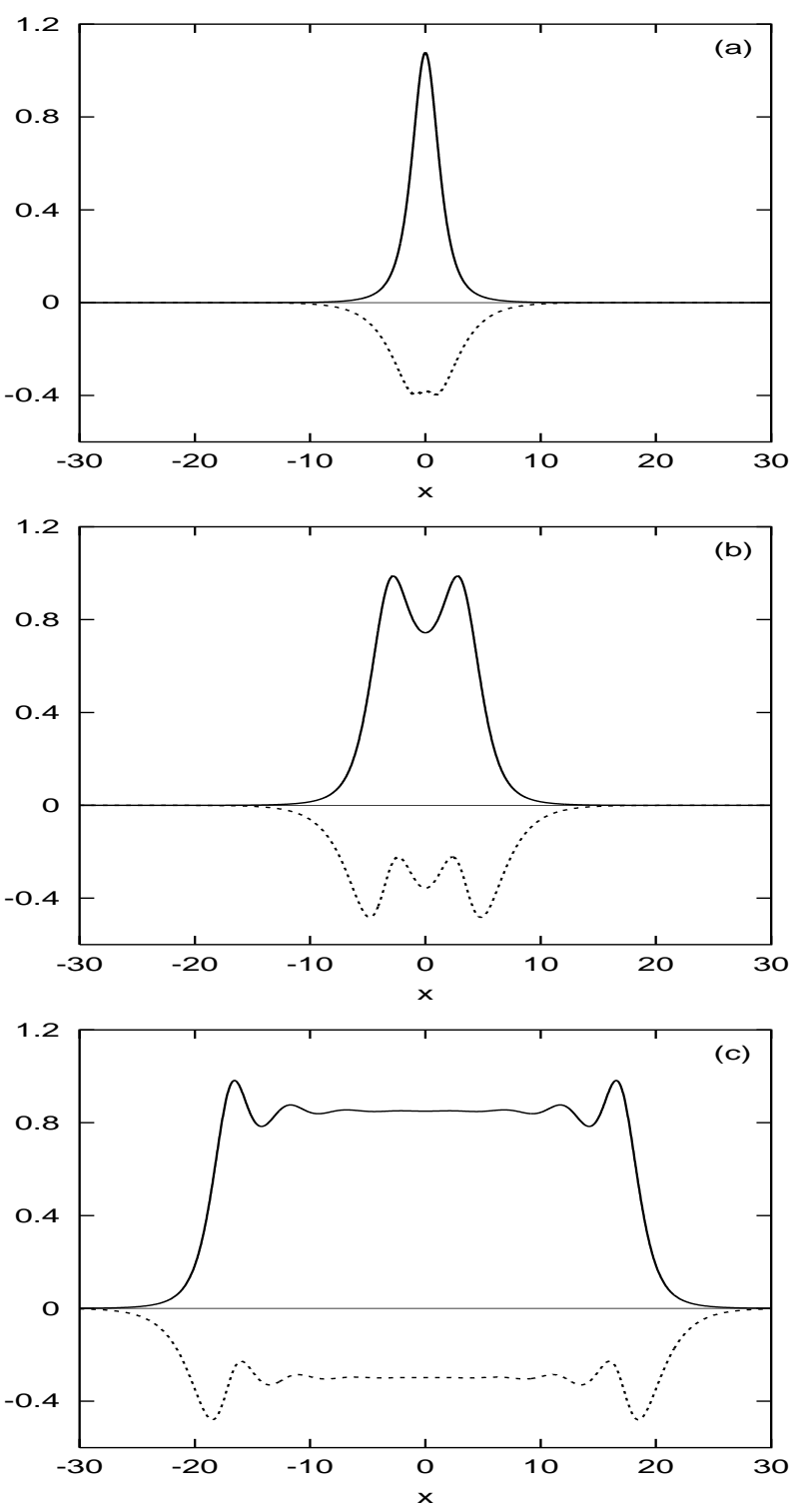

FIG. 2: (a),(b),(c): Solutions at the corresponding points in Fig 1 Solid line: real part; dashed line: imaginary part.

Despite the fact that negative values of the diffusivity $c$ are not physically meaningful, we did continue to $c<0-$ in the hope that the resulting branch would reach a turning point and then return to the positive $c$-semiaxis (which it did indeed do). The resulting branch is also shown in Fig 1 As we move into the region $c<0$, the $\psi_{+}$solution gradually develops into a three-humped state and when the curve returns to $c=0$, we have a complex of the $\psi_{(-+-)}$ type, with a large separation between the individual solitons. (This complex of three solitons of the parametrically driven nonlinear Schrödinger equation was previously found in Ref. 9 ].) Crossing into the $c>0$ region, the central $\psi_{+}$ soliton in the complex transforms as if it did not have the $\psi_{-}$solitons attached to its flanks. As a result, the $c>0$ portion of the corresponding $\|\psi(c)\|$ curve has virtually the same shape as the curve resulting from the continuation of $\psi_{+}$to the region $c>0$; the only difference is that the curve emanating out of $\psi_{(-+-)}$is shifted upwards relative to the curve emanating out of $\psi_{+}$. Similarly to the continuation of $\psi_{+}$, the continuation of $\psi_{(-+-)}$goes via a series of turning points, with each pass of the turning point resulting in the creation of another hump in the middle of the central region which becomes a long plateau. The lateral $\psi_{-}$solitons are not affected by this process. The linearised spectrum is the union of the spectrum of the long pulse described in the previous paragraph and spectra of two $\psi_{-}$ solitons. In particular, it includes two positive real eigenvalues contributed by the $\psi_{-}$'s and so the entire branch resulting from the continuation of $\psi_{(-+-)}$is unstable. We disregard it in what follows.

The plateau arising in the final stage of continuation of the two branches shown in Fig 1 is nothing but an interval 
on the $x$-axis where $\psi$ equals $\Psi_{+}^{(0)}$, the flat nonzero solution given by Eq.(15). The corresponding value of $c, c_{\text {lim }}=0.54$, falls within the region $c \geq c_{-}$where the background $\Psi_{+}^{(0)}$ is stable. Here $c_{-}(\gamma, h)$ is given by Eq. (10); in particular, $c_{-}(0.5,0.8)=0.224$. In the language of phase transitions, the long pulse shown in Fig[2](c) can be seen as a "bubble" of one stable phase in another one.

\section{The bound state interpretation}

The long pulse can also be interpreted as a bound state of two fronts interpolating between different stable backgrounds, $\psi=0$ and $\psi=\Psi_{+}^{(0)}$. This intuitively-appealing idea can be put on the quantitative footing by considering the spatial decay of perturbations to the flat background $\Psi_{+}^{(0)}$.

Indeed, consider the turning point separating the second stable branch from the first unstable branch in Fig 1 (This is a point with coordinates $c=0.37$ and $\|\psi\|=2.48$. Note that we are only considering branches obtained by the continuation of the soliton $\psi_{+}$to positive $c$. Branches obtained by the continuation of $\psi_{-}$to negative $c$ first, are disregarded here.) It is at this point that the modulus squared of $\psi(x)$ becomes double-humped; before that, that is on the branch that leads to this point (the first unstable branch), the function $|\psi(x)|^{2}$ remained single-humped despite the double-humped imaginary part. Letting $h=0.8, \gamma=0.5$ and $c=0.37$ we check that $4 A_{+}^{2}\left(A_{+}^{2}-1\right)>\gamma^{2}$ and hence, according to section IA the quadratic (9), (13) has two complex roots, $k^{2}=s_{r}+i s_{i}$ and $\left(k^{2}\right)^{*}$. Therefore the wavenumber $k$ is complex as well: $k=k_{r}+i k_{i}$. Solving equation (13) for $s$ we obtain, subsequently,

$$
k_{r}=\frac{1}{\sqrt{2}}\left(s_{r}+\sqrt{s_{r}^{2}+s_{i}^{2}}\right)^{1 / 2}=1.36, \quad k_{i}=\frac{s_{i}}{2 k_{r}}=0.19 .
$$

(We have chosen positive values for $k_{r}$ and $k_{i}$ here.)

For $c$ near the turning point in question, the plateau has not yet formed between the two humps and so they can be crudely thought of as two overlapping NLS-like solitons with oscillations on their adjacent (i.e. partner-facing) tails. The bound state arises when one solitary wave is trapped in the potential well formed by the oscillatory tail of its partner. Making use of the potential of interaction of two attractive NLS solitons [27], $U_{\text {eff }} \propto \exp \left(-k_{i} z\right) \cos \left(k_{r} z\right)$ (where $z$ stands for the distance between the two humps), and taking into account that $k_{i} / k_{r} \ll 1$, we obtain a rough estimate for the separation: $z=\pi / k_{r}$. This formula could also be derived from purely qualitative considerations; all one needs to notice is that the absolute value of the field $\psi$ obtained from the linear superposition of two in-phase solitons is maximised (and so the energy is minimised) by placing one soliton at the first maximum of $|\psi|$ on the tail of its partner. Substituting $k_{r}$ from (37), the approximate formula gives $z=2.31$. This is in qualitative agreement with the numerically found value $z=2.77$.

Moving further along the (second stable) branch, the plateau appears and the two humps can no longer be approximated by the NLS-like solitons. This makes the above estimate invalid. The solution can still be regarded as a bound state of two fronts but this time, in order to calculate the characteristic width of the pulse one would need to know the full profile of the front.

We conjecture that for a given $h$ and $\gamma$, a free-standing stationary front exists just for a single value of $c$, namely $c=c_{\text {lim }}$. (We are planning to verify this conjecture in future publications.) On the other hand, stable and unstable bound states of fronts exist in a finite interval of $c$ values containing $c=c_{\text {lim }}$ as an internal point. It is fitting to note here that similar pulse-to-front transformations occur also in the other system featuring the subcritical bifurcation, viz. the cubic-quintic Ginzburg-Landau equation with internal gain [28].

\section{THE $h$ VS $c$ DIAGRAM}

As we mentioned in the previous section, there is a certain discrepancy between the adiabatic analysis and numerical continuation. Numerically, the $\psi_{-}$soliton was found to be continuable all the way to $c=+\infty$ whereas the adiabatic approach predicted the existence of a turning point at $c=3 \tilde{c}_{2}$, where the $\psi_{-}$should have merged with the $\psi_{+}$branch. As for the $\psi_{+}$solution, we found that it turns into a pulse with the double-humped imaginary part (and not into the $\psi_{-}$branch as suggested by the adiabatic approximation.) In order to shed some light on the possible source of the discrepancy we performed the numerical continuation of the pulse $\psi_{-}$in $h$, for several fixed values of $c$. Here, by the $\psi_{-}$we mean the pulse solution which results from the continuation of the Schrödinger $\psi_{-}$soliton to positive $c$, for some fixed large value of $h$ (in our case for $h=0.8$.) Having obtained this starting-point solution for several values of $c$, we then continued it in $h$, from $h=0.8$ to smaller $h$. The stability of the arising solutions was examined by computing eigenvalues of the operator at sample values of $h$. 

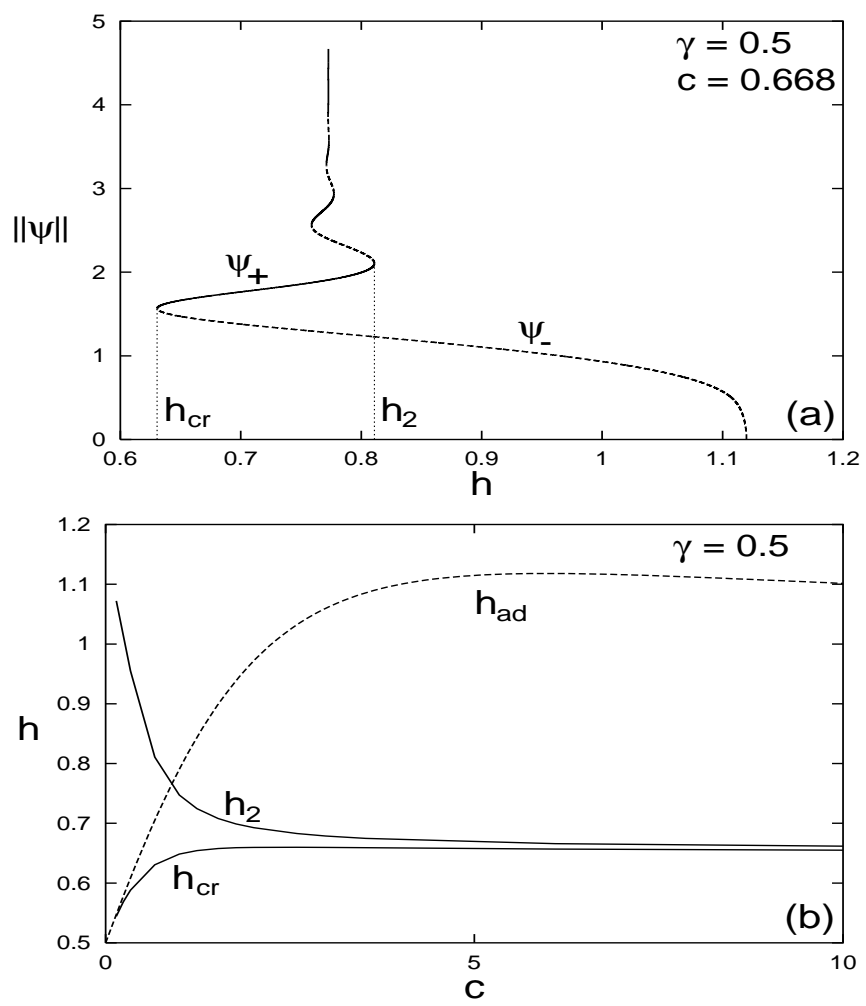

FIG. 3: (a) The Sobolev norm of the solitary wave solution as a function of the driving strength $h$. The solid and dashed lines indicate stable and unstable branches, respectively. (b) The pulse existence region on the $(c, h)$-plane. Two pulse solutions, $\psi_{+}$and $\psi_{-}$, are born as $h$ exceeds the value $h_{\mathrm{cr}}(c)$ depicted by the lower solid line. The upper solid curve gives the upper boundary of the $\psi_{+}$-pulse's existence domain, $h_{2}(c)$. Also shown is the adiabatic approximation to the saddle-node bifurcation curve, Eq.(33) (dashed line).

In each case considered, the $\psi_{-}$branch was found to turn into the $\psi_{+}$solution as $h$ reached the threshold value $h_{\mathrm{cr}}=h_{\mathrm{cr}}(\gamma, c)$. (That is, for $h>h_{\mathrm{cr}}$ there are two branches of solutions whereas for $h<h_{\mathrm{cr}}$, there is none; see Fig 3 (a)). The entire $\psi_{-}$branch is unstable; the single positive real eigenvalue moves to the negative semiaxis as the branch is continued past the turning point. Continuing the arising $\psi_{+}$branch to larger $h$, we reach another turning point at $h=h_{2}$, where the $\psi_{+}$solution transforms into a pulse with the double-humped imaginary part. The values $h_{\mathrm{cr}}$ and $h_{2}$ are shown in Fig[3(b), as functions of $c$ (for the fixed $\gamma=0.5$ ). As $c \rightarrow \infty$, the difference $h_{2}-h_{\mathrm{cr}}$ decreases but remains nonzero. We verified this by computing $h_{\mathrm{cr}}$ and $h_{2}$ for equation (36) which pertains to $c=\infty$. In the same plot we display the function (33) which gives the adiabatic approximation to the curve $h_{\mathrm{cr}}(c)$. (Note that for small $c$, there is a good agreement between numerical and approximate values but as $c$ grows, the two curves diverge.)

Continuing the $\psi_{+}$branch past the second turning point, the solution adds another hump in the middle of the pulse, turns back again, adds another one, and so on. (See Fig[3(a)). A long plateau developes in the middle of the pulse, just like when it was continued in $c$. Similarly to the $c$-continuation, the turning points on the $h$-axis separate regions of stability from regions of instability, with the instability being caused by a single positive real eigenvalue (which moves through $\lambda=0$ at the turning points.)

From figure 3(b) it is clear why the saddle-node bifurcation point where the $\psi_{+}$and $\psi_{-}$solutions would merge, did not appear in Fig 1. The reason is that Fig 1 was plotted for a relatively large value of $h(h=0.8)$ whereas according to Fig 3(b), a horisontal line $h=$ const with $h>0.660$ can have no intersections with the saddle-node curve $h_{\mathrm{cr}}(c)$. (Here all the numbers are for $\gamma=0.5$.) The same Fig 3 (b) explains what semed to be a discrepancy between the adiabatic analysis and the numerical continuation of the soliton $\psi_{-}$in $c$. The numerical result that seemed to contradict the adiabatics was that for $h=0.8, \psi_{-}$could be continued without bounds. It is now obvious from Fig 3 (b) that the unbounded continuation is only possible for $h$ greater than 0.660 . Continuing the $\psi_{-}$soliton to positive $c$ for $h$ smaller than 0.660 , the branch turns back (already as the $\psi_{+}$pulse) after hitting the lower solid curve in Fig 3 (b). Therefore, the pattern arising for $h$ close to $\gamma$ actually is in qualitative agreement with the adiabatic analysis, which was expected to be valid precisely for small $c$ or, equivalently, for small $h-\gamma$ differences. 


\section{CONCLUDING REMARKS}

In this paper we studied a cubic complex Ginzburg-Landau equation in which linear losses and diffusion are compensated by the linear parametric drive. The nonlinear term in the equation was taken to be purely conservative.

There are three stationary homogeneous solutions to equation (4), and we have shown that the $\psi=0$ solution is stable if $h \leq \sqrt{1+\gamma^{2}}$, as long as $c \geq 0$. This stability condition coincides with the corresponding condition for the nonlinear Schrödinger case (i.e. for $\bar{c}=0$ ). On the other hand, the stability properties of the nonzero homogeneous solutions are not the same as in the $c=0$ case. Indeed, unlike for $c=0$, there is a stable flat nonzero solution $\psi=\Psi_{+}^{(0)}$ for sufficiently large diffusion coefficients, $c \geq c_{-}(h, \gamma)$.

Having established the persistence of the NLS solitons $\psi_{+}$and $\psi_{-}$for small nonzero $c$, we continued them in $c$ numerically. The continuation of the soliton $\psi_{+}$yields a sequence of pulse-like solutions, separated by turning points, with increasing number of humps. The stability of these solutions changes at each turning point, so that stable multihump solutions coexist with unstable ones. It is fitting to note here that the multistability of multipulse solutions is not observed in the Schrödinger limit where only the two-soliton complex was found to be stable 9 . As $c \rightarrow c_{\lim }$, where $c_{\lim }=c_{\lim }(h, \gamma)$, the solution takes the form of a long plateau (an interval of the stable background $\left.\Psi_{+}^{(0)}\right)$ sandwiched between two fronts.

We also performed the continuation in $h$, for the fixed $c$. For each $c>0$, two localised solutions are born in a saddle-node bifurcation as $h$ exceeds a threshold value. (We obtained an analytic formula for the threshold in the adiabatic approximation; it is in agreement with the numerical results for small $c$.) The subsequent continuation gives rise to a sequence of coexisting stable multihump solutions culminating in a bound state of two widely separated fronts.

Solitary pulses in the form of long shelves (plateaus) can allow easy experimental observation in physical systems described by our model. In particular, they may be employed as a natural basis for the non-return-to-zero (NRZ) format of the data transmission in optical telecommunications. In the NRZ format, the 1 and 0 bits are coded, respectively, by sending or withholding the signal within a standard time slot.

A string of several 1's looks as a long uniform pulse of an essentially arbitrary length. The stability of such pulses is crucial to maintain the fixed shape of the long array of 1's, and to prevent the inter-symbol interference, i.e., the blurring of empty intervals between such strings, which represent (strings of) 0's (see, e.g. Ref. [29] and references therein.) In the case of lasers, which can also be described by the present model [20], the possibility of the generation of stable long pulses of arbitrary duration, i.e., an effective tunability of the output, is an essential advantage too.

\section{Acknowledgments}

We thank Nora Alexeeva for her advice on numerics. The first author's (I.B.'s) work was supported by the NRF of South Africa under grant No.2053723, by the Johnson Bequest Fund and the URC of the University of Cape Town. The second author (S.C.) was supported by the NRF and the Harry Crossley Foundation. B.A.M. appreciates hospitality of the Department of Physics at the Universität Erlangen-Nürnberg, and a partial support from the European Science Foundation, through the " $\pi$-shift" programme.

${ }^{\star}{ }^{*}$ Email address: igor@cenerentola.mth.uct.ac.za

[†] Email address: simon@light.mth.uct.ac.za

[§] Email address: malomed@eng.tau.ac.il

[1] M.C. Cross and P.C. Hohenberg, Rev. Mod. Phys. 65, 851 (1993).

[2] T. Bohr, M.J. Jensen, G. Paladin and A. Vulpiani. Dynamical Systems Approach to Turbulence. Cambridge University Press, 1998

[3] N. Akhmediev and A. Ankiewicz. Solitons. Nonlinear Pulses and Beams. Chapman and Hall, 1997; N. Akhmediev and A. Ankiewicz. Solitons of the Complex Ginzburg-Landau Equation. In: S. Trillo, W. Torruellas (Eds.) Spatial Solitons. Springer Series in Optical Sciences, vol. 82, 2001

[4] I.S. Aranson and L. Kramer, Rev. Mod. Phys. 74, 99 (2002)

[5] Proceedings of the Workshop on the Complex Ginzburg-Landau Equation: Theoretical Analysis and Experimental Applications in the Dynamics of Extended Systems. Edited by M. Bär and A. Torcini. Physica D 174, 1-220 (2003)

[6] B.A. Malomed, Physica D 29, 155 (1987)

[7] O. Thual and S. Fauve, J. Phys. (Paris) 49, 1829 (1988); S. Fauve and O. Thual, Phys. Rev. Lett. 64, 282 (1990)

[8] I.V. Barashenkov, M.M. Bogdan, V.I. Korobov, Europhys. Lett. 15, 113 (1991)

[9] I.V. Barashenkov and E.V. Zemlyanaya, Phys. Rev. Lett. 83, 2568 (1999) 
[10] M. Bondila, I.V. Barashenkov and M.M. Bogdan, Physica D 87, 314 (1995); S. Longhi, G. Steinmeyer, and W.S. Wong, J. Opt. Soc. Am. B 14, 2167 (1997); N.V. Alexeeva, I.V. Barashenkov and D.E. Pelinovsky, Nonlinearity 12, 103 (1999); V.S. Shchesnovich, I.V. Barashenkov, Physica D 164, 83 (2002)

[11] J.W. Miles, J. Fluid Mech. 148, 451 (1984)

[12] W. Zhang and J. Viñals, Phys. Rev. Lett. 74, 690 (1995); X. Wang and R. Wei, Phys. Rev. E 57, 2405 (1998)

[13] C. Elphick and E. Meron, Phys. Rev. A 40, 3226 (1989)

[14] B. Denardo, B. Galvin, A. Greenfield, A. Larraza, S. Putterman, and W. Wright, Phys. Rev. Lett. 68, 1731 (1992); S.-Y. Lou and G. Huang, Mod. Phys. Lett. B 9, 1231 (1995); G. Huang, S.-Y. Lou, and M. Velarde, Int. J. Bif. and Chaos 6, 1775 (1996); J. Yu, Phys. Lett. A 259, 366 (1999); N.V. Alexeeva, I.V. Barashenkov and G.P. Tsironis, Phys. Rev. Lett. 84, 3053 (2000); W. Chen, B. Hu, and H. Zhang, Phys. Rev. B 65, 13402 (2002)

[15] P. Coullet, Phys. Rev. Lett. 56, 724 (1986)

[16] T. Frisch, S. Rica, P. Coullet and J.M. Gilli, Phys. Rev. Lett. 72, 1471 (1994); T. Frisch, Physica D 84, 601 (1995)

[17] P. Coullet, J. Lega, and Y. Pomeau, Europhys. Lett. 15, 221 (1991)

[18] I.V. Barashenkov, S. Woodford, and E.V. Zemlyanaya, Phys. Rev. Lett. 90, 54103 (2003)

[19] A. Mecozzi, W.L. Kath, P. Kumar, and C.G. Goedde, Opt. Lett. 19, 2050 (1994); S. Longhi, Opt. Lett. 20, 695 (1995); K. Staliunas, J. Mod. Opt. 42, 1261 (1995); S. Longhi, J. Mod. Opt. 43, 1089 (1996); G.-L. Oppo, A.J. Scroggie, and W.J. Firth, Phys. Rev. E 63, 66209 (2001)

[20] S. Longhi, Opt. Lett. 21, 860 (1996); S. Longhi, Europhys. Lett. 37, 257 (1997)

[21] S.V. Kiyashko, L.N. Korzinov, M.I. Rabinovich, and L.S. Tsimring, Phys. Rev. E 54, 5037 (1996); L.S. Tsimring and I.S. Aronson, Phys. Rev. Lett. 79, 213 (1997)

[22] S. Longhi, Opt. Commun. 149, 335 (1998)

[23] P. Coullet, J. Lega, B. Houchmanzadeh, and J. Lajzerowicz, Phys. Rev. Lett. 63, 1352 (1990); P. Coullet and K. Emilsson, Physica D 61, 119 (1992); B.A. Malomed and A.A. Nepomnyashchy, Europhys. Lett. 27, 649 (1994); C. Elphick, A. Hagberg, B.A. Malomed and E. Meron, Phys. Lett. A 230, 33 (1997); C. Utzny, W. Zimmermann, and M. Bär, Europhys. Lett. 57, 113 (2002); G.J. de Valcárcel, I. Pérez-Arjona, and E. Roldán, Phys. Rev. Lett. 89, 164101 (2002)

[24] H. Sakaguchi and B. Malomed, J. Phys. Soc. Jpn. 72, 1360 (2003)

[25] S. Longhi, A. Geraci, Appl. Phys. Lett. 67, 3060 (1995)

[26] E.J. Doedel, X.J. Wang and T.F. Fairgrieve, AUTO 94: Software for continuation and bifurcation in ordinary differential equations, Applied Mathematics Reports, California Institute of Technology

[27] D. Cai, A.R. Bishop, N. Grønbech-Jensen, and B.A. Malomed, Phys. Rev. E 49, 1677 (1994)

[28] V. Hakim, P. Jakobsen, and Y. Pomeau, Europhys. Lett. 11, 19 (1990); B.A. Malomed and A.A. Nepomnyashchy, Phys. Rev. A 42, 6009 (1990)

[29] R. Driben, B.A. Malomed, M. Gutin and U. Mahlab, Opt. Commun. 218, 93 (2003). 